\title{
A Novel Assay Reveals a Maturation Process during Ascospore Wall Formation
}

\author{
Kai Zhang ${ }^{\dagger}$, Leor Needleman ${ }^{\dagger}$, Sai Zhou ${ }^{\dagger}$ and Aaron M. Neiman * \\ Department of Biochemistry and Cell Biology, Stony Brook University, Stony Brook, NY 11794-5215, USA; \\ zkb422@hotmail.com (K.Z.); leorneedleman@gmail.com (L.N.); zs1001365@gmail.com (S.Z.) \\ * Correspondence: aaron.neiman@stonybrook.edu; Tel.: + 1-631-632-1543; Fax: + 1-631-632-8575 \\ † These authors contributed equally to this work.
}

Received: 12 September 2017; Accepted: 29 September 2017; Published: 2 October 2017

\begin{abstract}
The ascospore wall of the budding yeast Saccharomyces cerevisiae consists of inner layers of similar composition to the vegetative cell wall and outer layers made of spore-specific components that confer increased stress resistance on the spore. The primary constituents of the outer spore wall are chitosan, dityrosine, and a third component termed Chi that has been identified by spectrometry but whose chemical structure is not known. The lipophilic dye monodansylpentane readily stains lipid droplets inside of newly formed ascospores but, over the course of several days, the spores become impermeable to the dye. The generation of this permeability barrier requires the chitosan layer, but not dityrosine layer, of the spore wall. Screening of a set of mutants with different outer spore wall defects reveals that impermeability to the dye requires not just the presence of chitosan, but another factor as well, possibly Chi, and suggests that the OSW2 gene product is required for synthesis of this factor. Testing of mutants that block synthesis of specific aromatic amino acids indicates that de novo synthesis of tyrosine contributes not only to formation of the dityrosine layer but to impermeability of the wall as well, suggesting a second role for aromatic amino acids in spore wall synthesis.
\end{abstract}

Keywords: ascospore; spore wall; dityrosine; chitosan; tyrosine; sporulation

\section{Introduction}

The formation of ascospores is a defining feature of ascomycete fungi [1]. Ascospores are formed after meiosis and are surrounded by a specialized cell wall, hereafter referred to as the spore wall, which is distinct in both composition and morphology from the cell wall of vegetative cells. The best characterized ascospore wall is that of the baker's yeast Saccharomyces cerevisiae [2].

In response to nitrogen starvation in the presence of a poor carbon source, diploids of S. cerevisiae exit the mitotic cell cycle, undergo meiosis to generate four haploid nuclei and envelope each of those nuclei inside of newly formed plasma membranes to create four spores [2]. Each spore is surrounded by a spore wall and all four spores are contained within the remnant of the mother cell, the ascus. The spore wall is a more extensive structure than the vegetative cell wall and consists of inner layers of mannoproteins and $\beta$-glucans, which are similar in composition to the vegetative cell wall, and outer layers consisting of spore-specific components [3-5]. The primary component of the outer spore wall is the $\beta-1,4$-glucosamine polymer, chitosan [3]. This is synthesized through the action of the sporulation-specific deacetylases Cda1 and Cda2 on chitin produced by the chitin synthase Chs3 [6-8]. The chitosan is assembled into a visible layer outside of the mannan and $\beta$-glucan and then a layer consisting primarily of the cross-linked di-amino acid dityrosine is formed outside of the chitosan $[9,10]$. Chitosan is essential for assembly of the outer spore wall, so mutants in CHS3 lack both the chitosan and dityrosine layers [8]. 
Dityrosine is synthesized in the spore cytoplasm by the serial action of two enzymes, Dit1 and Dit2 and then exported to the spore wall through a transporter, Dtr1, in the spore plasma membrane [11,12]. Once exported, the dityrosine is assembled into a large polymer on the surface of the spore wall, although the structure of the polymer built from the dityrosine has not been determined [9]. The dityrosine polymer is a polyaromatic compound and may serve an analogous role to the melanin found in some fungal cell walls [13]. Additionally, nuclear magnetic resonance (NMR) studies of isolated outer spore walls reveal the presence of another component, distinct from both chitosan and dityrosine, termed Chi [14]. The chemical nature of Chi is unclear, though its presence is independent of dityrosine and it may serve to help connect the dityrosine and chitosan layers [14].

These unique outer spore wall components are the distinguishing feature of ascopores and confer on the spore increased resistance to a wide variety of environmental insults including high salt, temperature, low and high $\mathrm{pH}$, as well as digestion in the gut of insects [15]. Spores are also more resistant to exposure to organic solvents such as diethyl ether, and ether exposure is commonly used to test for spore wall integrity [16]. How the spore wall confers resistance to such a variety of insults is not clear. It is likely that different components of the spore wall contribute resistance to different agents. For example, the dityrosine layer creates a barrier to the diffusion of proteins in and out of the spore wall and so dit1 $\Delta$ mutants are sensitive to treatment with glucanase enzymes; however, dit $1 \Delta$ mutants do not display strong sensitivity to ether vapor [14,17].

In addition to the genes encoding enzymes responsible for chitosan and dityrosine synthesis, several additional genes important for outer spore wall assembly have been identified $[12,14,17,18]$. Mutations in some of these genes lead to reduced levels of Chi and dityrosine in the spore wall, some show only reduced dityrosine, while others display increased sensitivity to environmental challenge but lack obvious spore wall defects $[14,17,18]$. In most cases, it is not clear how the gene products involved promote spore wall assembly.

Staining of spores with the hydrophobic dye monodansylpentane (MDH) revealed that wild-type spores become impermeable to the dye over the course of a few days after spore formation. This maturation requires the chitosan layer of the outer spore wall, but not the dityrosine layer. A set of previously identified spore wall mutants was assayed for MDH permeability. The results of these tests distinguish between mutants that specifically affect dityrosine synthesis and assembly versus those with broader effects on the outer spore wall. Mutants defective in aromatic amino acid biosynthesis were also examined for effects on spore wall formation. The results reveal that de novo tyrosine synthesis contributes to the formation of dityrosine layer precursors. In addition, tyrosine is important for spore wall maturation independently of its role as a dityrosine precursor, suggesting that derivatives of tyrosine contribute to other aspects of spore wall structure.

\section{Materials and Methods}

\subsection{Yeast Strains and Plasmids}

Standard media were used unless otherwise noted [19]. The strains used in this study are listed in Table 1. The integrating plasmid pRS304 [20] was used to introduce TRP1 into strains in the AN120 background. The pRS304-PET10-RFP plasmid was constructed by PCR amplification of a PET10-GFP fusion from the GFP-tagged yeast collection strain [21] from -700bp upstream of the PET10 start codon to the $3^{\prime}$ end of the CYC1 terminator. This PCR fragment was digested with BglII and ClaI sites, for which sites were introduced at the $5^{\prime}$ and $3^{\prime}$ ends respectively, and cloned into BamHI/ClaI cut pRS304. This plasmid was then cut with PacI and AscI to release the GFP coding region and the vector backbone ligated with a PCR fragment carrying the RFP coding region and PacI and AscI digested ends. Haploids AN117-4B and AN117-16D were transformed with EcoRI digested pRS304-PET10-RFP to target integration of the plasmid to the PET10 promoter region. The resulting haploids, yKZ72 and yKZ70, respectively, were mated to generate yKZ104. Homozygous deletions of DIT2 and OSW2 were generated by PCR-mediated replacement with the kanamycin and hygromycin resistance markers, 
respectively [22,23], in both AN117-4B and AN117-16D and mating of the resulting haploids to generate ySZ233 and ySZ265. The diploids yKZ108 and yKZ110 were generated by deleting PHA2 and $T Y R 1$, respectively, with the kanamycin marker in the same manner, with the additional step that the resulting diploids were then transformed with Bsu36I digested pRS304. The diploids yKZ107, yKZ111 and yKZ112 were made by transformation of AN120, AN262, and AN264, respectively, with Bsu36I digested pRS304.

Table 1. Strains used in this study.

\begin{tabular}{|c|c|c|}
\hline Strain & Genotype & Source \\
\hline AN120 & 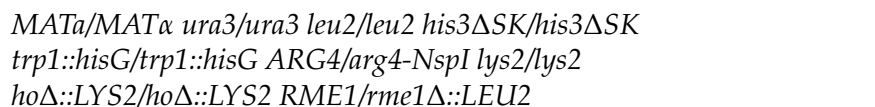 & [24] \\
\hline AN117-4B & 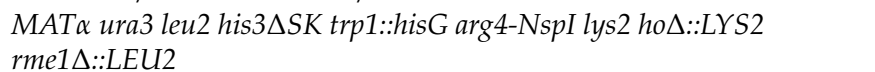 & [24] \\
\hline AN117-16D & 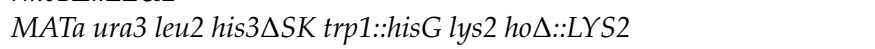 & [24] \\
\hline yKZ70 & as AN117-16D plus PET10::PET10-RFP::TRP1 & this study \\
\hline yKZ72 & as AN117-4B plus PET10::PET10-RFP::TRP1 & this study \\
\hline yKZ104 & as AN120, plus PET10::PET10-RFP::TRP1/PET10::PET10-RFP::TRP1 & this study \\
\hline AN262 & as AN120, plus chs3 $3:: H I S 3 M X 6 /$ chs $3 \Delta:: H I S 3 M X 6$ & [25] \\
\hline AN264 & 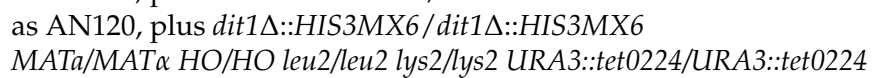 & [25] \\
\hline CL6 & $\begin{array}{l}l d s 1 \Delta:: H I S 3 M X 6 / l d s 1 \Delta:: H I S 3 M X 6 \text { rrt8 } \Delta:: k a n M X 6 / r r t 8 \Delta:: k a n M X 6 \\
l d s 2 \Delta:: h p h M X 6 / l d s 2 \Delta:: h p h M X 6\end{array}$ & [14] \\
\hline CL7 & 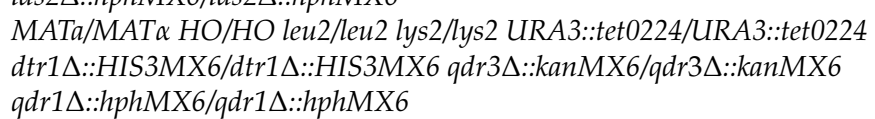 & [14] \\
\hline CL15 & 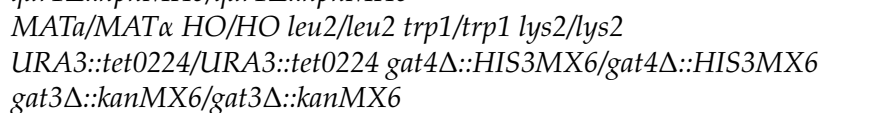 & [14] \\
\hline CL26 & 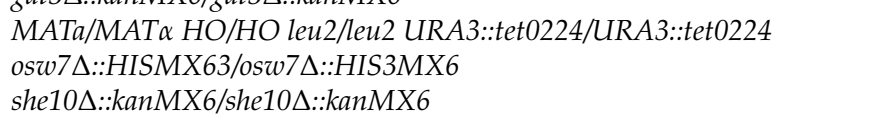 & [14] \\
\hline CL35 & 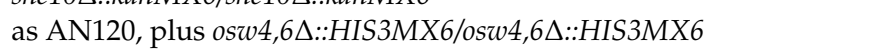 & [14] \\
\hline CL57 & 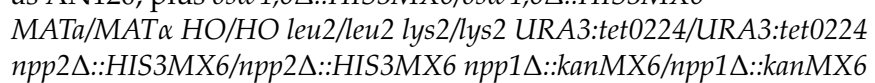 & {$[14]$} \\
\hline ySZ233 & as AN120, plus dit2 $2:: k a n M X 6 /$ dit2 $2:: k a n M X 6$ & this study \\
\hline ySZ265 & $\begin{array}{l}\text { as AN120, plus osw2 } 2:: \text { hphMX4/osw2 } 2 h p h M X 4 \\
\text { MATa/MATa HO/HO his3/his3 trp1/trp1 lys2/lys2 }\end{array}$ & this study \\
\hline MYA-1810 & $\begin{array}{l}\text { LEU2::P URA3-tetR-GFP/LEU2::PURA3-tetR-GFP } \\
\text { REC8::HA3-URA3/REC8::HA3-URA3 } \\
\text { URA3::tetO224/URA3::tetO224 dtr1 }:: H I S 3 M X 6 / d \operatorname{tr} 1 \Delta:: H I S 3 M X 6 \\
\text { MATa/MATa HO/HO his3/his3 trp1/trp1 lys2/lys2 }\end{array}$ & [26] \\
\hline MYA-1824 & $\begin{array}{l}\text { LEU2::: } P_{\text {URA3-tetR-GFP/LEU2::P }} \text { URA3-tetR-GFP } \\
\text { REC8::HA3-URA3/REC8::HA3-URA3 } \\
\text { URA3::tetO224/URA3::tetO224 osw3A::HIS3MX6/osw3A::HIS3MX6 } \\
\text { MATa/MATa HO/HO his3/his3 trp1/trp1 lys2/lys2 }\end{array}$ & [26] \\
\hline MYA-2022 & $\begin{array}{l}\text { LEU2:::PURA3-tetR-GFP/LEU2::PURA3-tetR-GFP } \\
\text { REC8::HA3-URA3/REC8::HA3-URA3 } \\
\text { URA3::tetO224/URA3::tetO224 osw5 }:: H I S 3 M X 6 / \text { osw5 }:: H I S 3 M X 6\end{array}$ & [26] \\
\hline yKZ107 & as AN120, plus trp1::hisG::TRP1/trp1::hisG & this study \\
\hline yKZ108 & $\begin{array}{l}\text { as AN120, plus pha2 } \triangle:: k a n M X 6 / \text { pha2 } \triangle:: k a n M X 6 \\
\text { trp1::hisG::TRP1/trp1::hisG }\end{array}$ & this study \\
\hline yKZ110 & $\begin{array}{l}\text { as AN120, plus tyr1 }:: \text { kanMX6/tyr1 }:: \text { kanMX6 } \\
\text { trp1::hisG::TRP1/trp1::hisG }\end{array}$ & this study \\
\hline yZK111 & as AN262, plus trp1::hisG::TRP1/trp1::hisG & this study \\
\hline yKZ112 & as AN264, plus trp1::hisG::TRP1/trp1::hisG & this study \\
\hline
\end{tabular}

\subsection{MDH Staining Assay}

Sporulation was performed by replica plating of patches grown on yeast extract peptone dextrose (YPD) medium to SPO plates ( $2 \%$ agar, $1 \%$ KOAc, $0.05 \%$ yeast extract, $0.05 \%$ amino acid mixture 
without arginine, $0.05 \%$ glucose) and incubation of the plates at $23{ }^{\circ} \mathrm{C}$. For staining of cells with MDH, cells were scraped off the plate with a toothpick and resuspended in 100 microliters of $2 \%$ KOAc. One microliter of a $5 \mathrm{mM}$ solution of monodansylpentane (Abgent, San Diego, CA, USA) dissolved in DMSO was added to the suspension and the cells were incubated for $5 \mathrm{~min}$ at $23^{\circ} \mathrm{C}$. Cells were pelleted, washed twice with 200 microliters of $2 \%$ KOAc, resuspended in 20 microliters of $2 \%$ KOAc and then 1.5 microliters transferred to microscopy slides for inspection.

\subsection{Dityrosine Fluorescence Assay}

Cells were sporulated in liquid culture. Overnight cultures were grown to saturation at $30{ }^{\circ} \mathrm{C}$ in YPD medium and then diluted into yeast extract peptone acetate (YPA) medium for $12 \mathrm{~h}$. The $\mathrm{OD}_{660}$ of these cells were measured, the cells pelleted, washed once in $2 \%$ KOAc and then resuspended in KOAc at an $\mathrm{OD}_{660}$ of 1.2. Cells were incubated in $2 \%$ KOAc for $48 \mathrm{~h}$ and then spores examined by fluorescence microscopy. The dityrosine fluorescence intensity was calculated for each spore by taking the average intensity at two points in the spore wall and subtracting the background fluorescence outside of the spore.

\subsection{Microscopy}

For the lipid droplet assay, stained cells were examined on a Zeiss Axioplan 2 microscope (Zeiss, Thornwood, NY, USA) using a blue fluorescence filter set (ex. $360 \mathrm{~nm}$, em. $460 \mathrm{~nm}$ ) for MDH signal and a red fluorescence set (ex. $540 \mathrm{~nm}$, em. $605 \mathrm{~nm}$ ) for the Pet10-RFP signal and DIC optics for transmitted light. Images were obtained using Zeiss Axiovision software. For the dityrosine assay, images were collected on a Zeiss Observer Z1 microscope using a dityrosine-optimized filter set (ex. $330 \mathrm{~nm}, \mathrm{em} .410 \mathrm{~nm}$ ) and a fixed $2 \mathrm{~s}$ exposure time for all images. Fluorescence intensity was quantified using Zeiss Zen software.

\subsection{Ether Tests}

Strains were sporulated in liquid as for the dityrosine assay. After $48 \mathrm{~h}$ in $2 \%$ KOAc, five-fold serial dilutions of sporulated cells were spotted on two YPD plates in glass petri dishes. One plate served as a plating control and the other was inverted for $30 \mathrm{~min}$ over filter paper (Whatman, Maidstone, United Kingdom) that had been soaked with $6 \mathrm{~mL}$ of di-ethyl ether (J.T. Baker, Phillipsburg, NJ, USA). Both plates were incubated at $30{ }^{\circ} \mathrm{C}$ for two days before photographing.

\section{Results}

\subsection{Staining with Monodansylpentane Reveals a Maturation Process during Spore Wall Development}

The lipophilic dye monodansylpentane (MDH) can be used to specifically label lipid droplets in yeast [27]. Earlier work revealed that lipid droplets separate into two populations during sporulation with some segregating inside the forming spore and others remaining outside the spore and associating with the spore wall as it forms $[5,14,28]$. This latter population of droplets disappears during spore wall assembly so that in newly formed asci, bright MDH staining is predominantly seen inside of the spores ([28]; Figure 1A). By contrast, when 8-day old asci were similarly stained with MDH, the dye was mainly visible in the ascal space between the spores and the majority of spores failed to show internal staining or showed only faint internal staining (Figure 1A). 


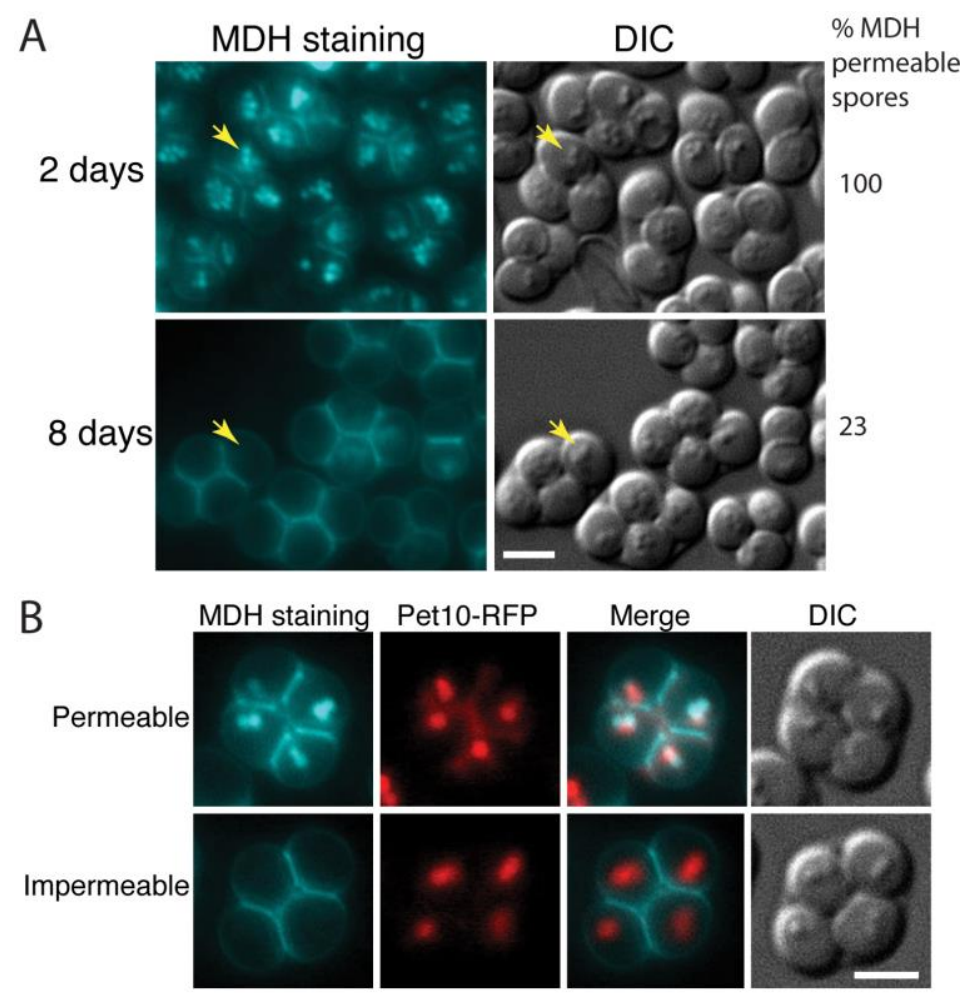

Figure 1. Older spores become impermeable to MDH. (A) Asci formed by the wild-type (AN120) strain after incubation on SPO plates for two days or eight days were stained with MDH and observed by fluorescence (blue staining) and light microscopy. The percentage of spores in the culture that displayed internal MDH staining is given at right (at least 500 spores counted). Yellow arrows indicate examples of lipid droplets visible by differential interference contrast (DIC) microscopy. Scale bar $=3$ microns. (B) Five-day old asci of a wild-type strain expressing the lipid droplet marker Pet10-RFP (yKZ104) were stained with $\mathrm{MDH}$ and examined by fluorescence microscopy. Examples of asci containing MDH permeable or impermeable spores are shown. Scale bar $=3$ microns.

This loss of internal MDH staining might indicate the consumption of neutral lipids and disappearance of droplets within the spores. However, apparent lipid droplets can still be seen as small 'bumps' in differential interference contrast (DIC) optics in the spores from both time points (Figure 1A). To confirm that lipid droplets are still present in the older spores, asci of a strain expressing a fluorescently tagged lipid droplet protein, Pet10-RFP, were examined by MDH staining five days after transfer to SPO medium (Figure 1B). In asci with spores that were permeable to the dye, the Pet10-RFP and MDH staining colocalized, as expected. In spores that were impermeable to $\mathrm{MDH}$, the localization of Pet10-RFP into discrete foci coincident with the lipid droplets seen in DIC was unchanged. This suggests that the lack of MDH staining in older spores is due to changes in permeability of the spores over time and not disappearance of the lipid droplets within spores.

If the loss of staining is due to changes in permeability of the spores, the spore wall is likely responsible. A dit1 $\Delta$ mutant strain, lacking the dityrosine layer and a chs $3 \Delta$ mutant strain, which lacks both the chitosan and dityrosine layers [8,29], were examined by MDH staining (Figure 2A). After eight days on SPO medium, the dit1 $\triangle$ spores predominantly lacked MDH staining, similar to the wild-type. By contrast, older spores in chs $3 \Delta$ strain remained as permeable to the dye as newly formed spores. To examine this process more closely, a time course analysis was performed, staining spores of wild-type, chs $3 \Delta$ and dit1 $\Delta$ strains with $\mathrm{MDH}$ at intervals over an eight day period (Figure 2B). This analysis revealed that the loss of staining was progressive; over the eight days, the fraction of spores in wild-type displaying intracellular MDH staining diminished from $100 \%$ to less than $30 \%$. This change in staining pattern was transient, as spores aged beyond 10 days displayed 
increasing permeability with time (unpublished obs.). The dit $1 \Delta$ mutant displayed similar kinetics to wild-type cells, with most spores becoming impermeable to the dye after eight days. By contrast, spores in the chs $3 \Delta$ mutant remained almost $100 \%$ permeable even after eight days of incubation. These results indicate that some maturation process occurring in the outer spore wall is responsible for impermeability to MDH staining and impermeability to the dye requires the proper assembly of the chitosan layer. However, the dityrosine layer of the spore wall is dispensable for this process.
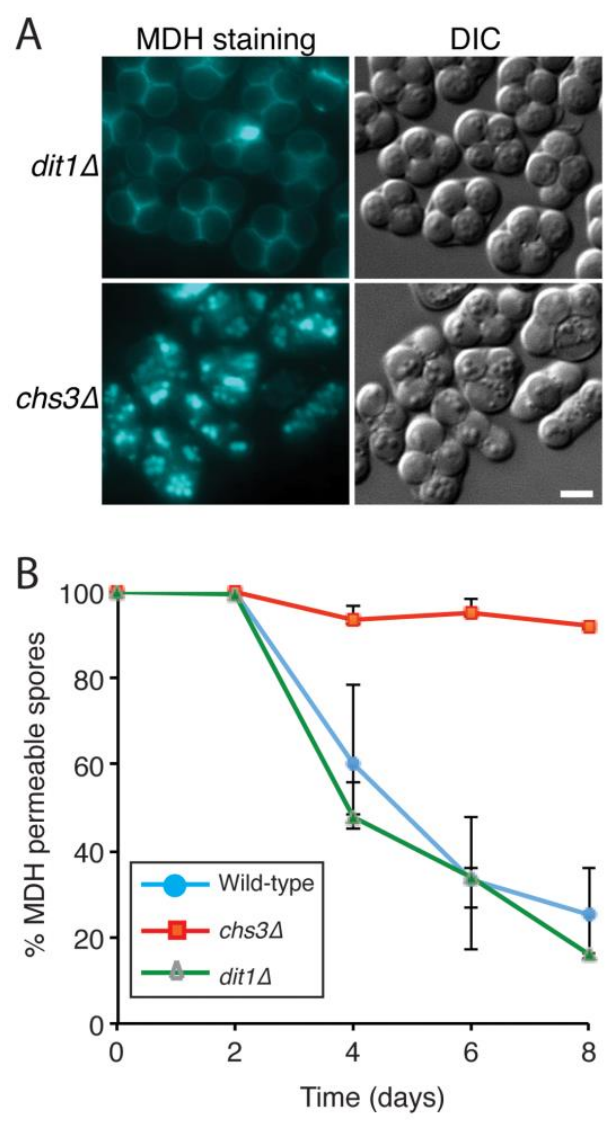

Figure 2. The outer spore wall is required for impermeability to $\mathrm{MDH}$. (A) Asci formed by dit1 $\Delta$ (AN264) or chs3 $\Delta$ (AN262) diploids after incubation on SPO plates for eight days were stained with $\mathrm{MDH}$ and observed by fluorescence (blue staining) and light microscopy. Scale bar $=3$ microns. (B) Time course of MDH staining wild-type (AN120), dit1 $\Delta$ (AN264), and chs3 $\Delta$ (AN262) strains were sporulated on SPO plates and at two day intervals, spores were stained with $\mathrm{MDH}$ and the percentage of spores showing MDH staining of lipid droplets within the spore was determined. 500 spores were scored at each time point for each strain. Error bars indicate the range of values obtained at each time point over three separate experiments.

\subsection{Acquisition of Impermeability Discriminates between Mutants with Outer Spore Wall Defects}

Chitosan is required for the maturation process but dityrosine is not. This could indicate either that chitosan itself is the source of the impermeability or that some non-dityrosine component of the outer wall that is dependent on chitosan for assembly is responsible. A number of mutants have been described that alter the outer spore wall without obviously altering the chitosan layer [14,18]. Genes reported to be important for dityrosine layer formation include DIT2 and DTR1 involved in dityrosine synthesis and export, respectively, and OSW3 and OSW5, which were identified on the basis that spore walls in mutants of these genes lack a barrier to the diffusion of protein-sized molecules out of the spore wall, similar to dit $1 \Delta$ mutants $[12,17,29]$. The osw $3 \Delta$ mutant, in particular, displays reduced dityrosine fluorescence from the wall. Furthermore, double mutants in the paralogous genes OSW4 
OSW6, OSW7 SHE10, and NPP1 NPP2, were all characterized by NMR analysis of their spore walls as lacking dityrosine but retaining both chitosan and Chi [14]. Three additional knockouts of paralogous genes LDS1 LDS2 RRT8, QDR1 QDR3 DTR1, and GAT3 GAT4 displayed defects in both dityrosine and Chi [14]. Finally, the OSW2 gene was identified as a strain that produces spores highly sensitive to ether vapor, suggesting an outer spore wall defect, but displaying no obvious defects or loss of spore wall components [18].

All of these mutants were examined for MDH staining after eight days of sporulation and the percentage of $\mathrm{MDH}$ permeable spores was determined (Figure 3). Of the genes previously implicated as involved in dityrosine layer assembly, spores in strains carrying mutations in DIT2, DTR1, and OSW5 all became largely impermeable to $\mathrm{MDH}$, similar to the wild-type and dit1 $\Delta$ strains, consistent with these genes being primarily involved in dityrosine layer formation. The $0 s w 4 \Delta \operatorname{osw} 6 \Delta$, osw $7 \Delta$ she $10 \Delta$, and $\operatorname{ssw} 3 \Delta$ strains all displayed intermediate levels of permeability between the wild-type and $\operatorname{chs} 3 \Delta$ controls. This increase in permeability was significantly different from wild-type ( $p<0.001$, student's $t$-test). The $n p p 1 \Delta n p p 2 \Delta$ mutant was as permeable as a chs $3 \Delta$ mutant strain. These results indicate that OSW3, OSW4/OSW6, OSW7/SHE10 and particularly NPP1/NPP2 have effects on the outer spore wall beyond dityrosine assembly. All of the mutants shown to have effects on the presence of Chi in the spore wall $(l d s 1 \Delta l d s 2 \Delta r r t 8 \Delta, q d r 1 \Delta q d r 3 \Delta d t r 1 \Delta$, gat $3 \Delta$ gat $4 \Delta)$ remained completely permeable to $\mathrm{MDH}$. As all of these mutants retain the chitosan layer [14]; this indicates that the presence of chitosan alone is not responsible for the impermeability of the spore wall to $\mathrm{MDH}$ and implicates Chi as important for this property of the wall.

Mutations in chs3 $\Delta$, but not dit1 $\Delta$ are also particularly sensitive to ether vapor [14], paralleling their effects on permeability to MDH. The osw $2 \Delta$ mutant, whose primary phenotype is sensitivity to ether vapor, remained permeable to the dye. This further extends the correlation between permeability and ether sensitivity and suggests that OSW2 is specifically involved in the synthesis of whatever spore wall component is responsible for impermeability to $\mathrm{MDH}$.

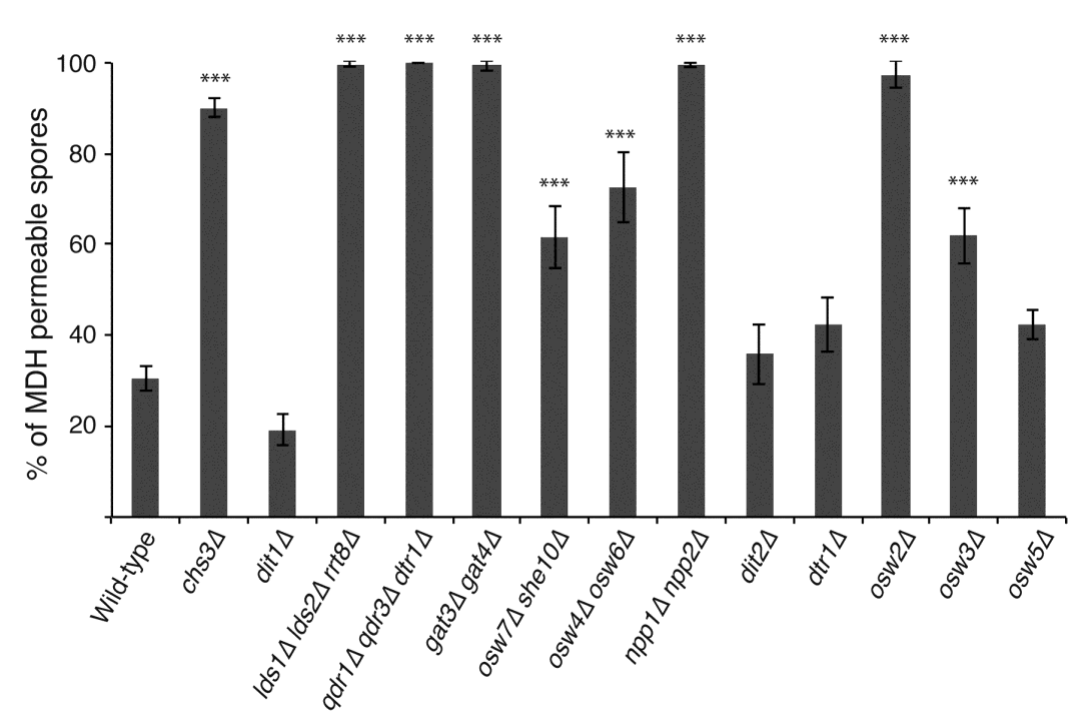

Figure 3. The effects of different outer spore wall mutants on MDH permeability. Wild-type (AN120),

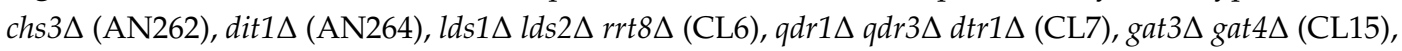
osw7 $\operatorname{she} 10$ (CL26), osw $4 \Delta$ osw6 $\Delta$ (CL35), npp1 1 npp $2 \Delta$ (CL57), dit2 $\Delta$ (ySZ233), dtr1 $($ MYA-1810), osw2 $\Delta$ (ySZ265), osw3 (MYA-1824), and osw5 (MYA-2022) diploids were incubated on SPO plates for eight days and then tested for MDH staining of lipid droplets within the spores. The percentage of spores displaying internal MDH staining is shown. Values are the averages from four experiments with at least 200 spores scored for each strain in each experiment. Error bars indicate one standard deviation. Asterisks denote strains in which permeability is significantly different from the wild-type $(p<0.001$ student's $t$-test). 
3.3. De Novo Synthesis of Tyrosine Contributes to Dityrosine Layer Assembly and to Additional Properties of the Spore Wall

Metabolomic studies of sporulating yeast cells indicate that, as cells progress through sporulation, there is an influx of carbon into pathways that lead to synthesis of spore wall precursors, both nucleotide sugars and aromatic amino acids [30]. The increased flux into the aromatic amino acid pathway was suggested to indicate synthesis of tyrosine for incorporation into the dityrosine layer of the spore wall. However, aromatic amino acids share common synthesis steps and so these data might also indicate a need for phenylalanine or tryptophan in the synthesis of some spore wall component. To test this directly, strains carrying specific defects in tryptophan $(\operatorname{trp} 1 \Delta)$, phenylalanine (pha2 $\Delta$ ) or tyrosine (tyr $1 \Delta)$ synthesis were sporulated and the spores examined. All three strains efficiently produced visible spores, indicating de novo synthesis of these amino acids is not essential for spore formation. Dityrosine fluorescence from the spore walls of each mutant was then quantified by fluorescence microscopy and compared to wild-type and dit $1 \Delta$ strains as controls (Figure $4 \mathrm{~A}$ ). While the $\operatorname{trp} 1 \Delta$ and pha2 $\Delta$ mutants display levels of dityrosine fluorescence comparable to wild-type, tyr $1 \Delta$ mutants display significantly reduced fluorescence, though not down to the background level seen in dit1 $\Delta$. These results indicate that de novo tyrosine synthesis contributes to formation of the dityrosine layer.
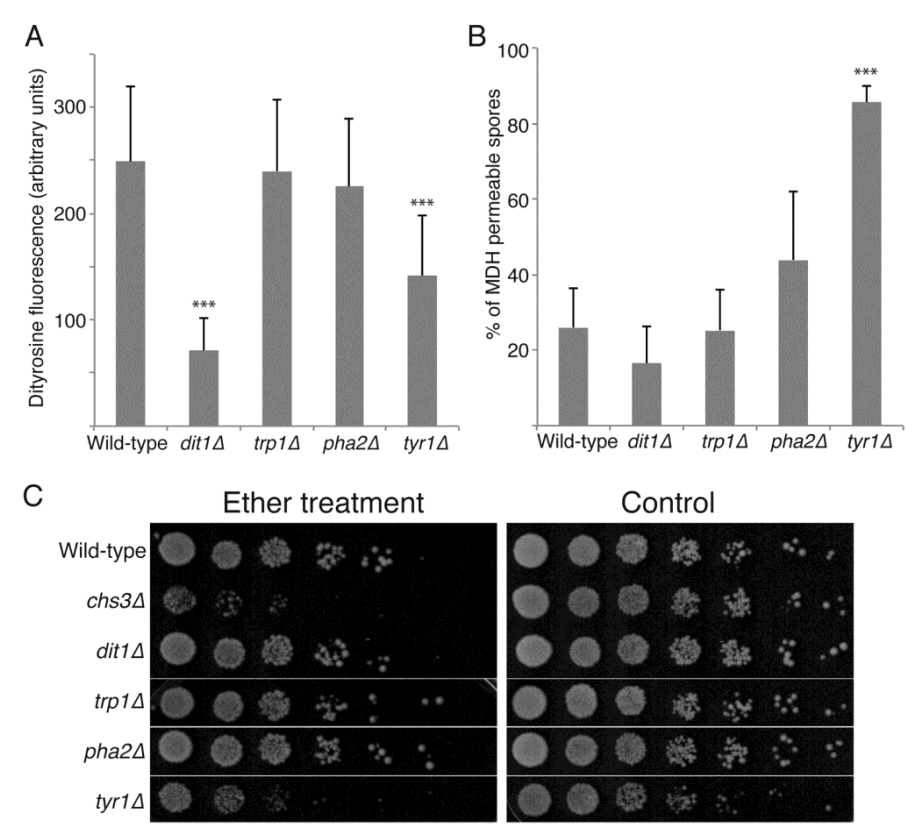

Figure 4. The effect of mutations in aromatic amino acid biosynthesis on outer spore wall formation.

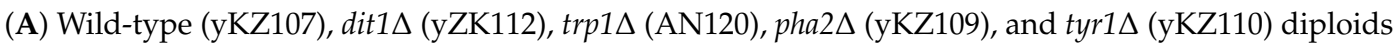
were sporulated for $48 \mathrm{~h}$ in liquid culture and the intensity of dityrosine fluorescence in the spore walls was measured by fluorescence microscopy. The average intensity from 20 different spores from each strain is shown. Error bars indicate one standard deviation. Asterisks indicate a significant change from the wild-type ( $p<0.001$ student's $t$-test). The experiment was performed three times with similar results; one representative experiment is shown. (B) The same strains as in (A) were examined by MDH staining after eight days incubation on solid SPO medium. The percentage of spores displaying internal MDH staining is shown. Values are the averages from three experiments with 500 spores scored for each strain in each experiment. Error bars indicate one standard deviation. Asterisks denote significant difference from the wild-type ( $p<0.001$ student's $t$-test). (C) Test of ether resistance. The same diploids as in (A) plus a chs3 $\Delta$ diploid (yZK111) were sporulated in liquid culture for $48 \mathrm{~h}$. Fivefold serial dilutions of each culture were spotted onto two rich medium (YPD) plates and then one plate (left panel) was exposed to ether vapor for 30 minutes. Both plates were incubated at $30{ }^{\circ} \mathrm{C}$ for two days before being photographed. 
The mutants in aromatic amino acid synthesis were further examined for spore wall defects by MDH staining of 8 -day old spores and by ether test (Figure $4 \mathrm{~B}, \mathrm{C}$ ). The pha $2 \Delta$ and $\operatorname{trp} 1 \Delta$ spores displayed decreased permeability to MDH similar to wild-type and were resistant to exposure to ether vapor. The tyr $1 \Delta$ mutant, however, displayed increased permeability of the spores and increased ether sensitivity. These phenotypes were stronger than those in the dit1 $\Delta$ mutant, which completely lacks dityrosine. Thus, the use of the MDH staining reveals that the biosynthesis of tyrosine plays an additional role in spore wall assembly beyond simply acting as a precursor for dityrosine synthesis.

\section{Discussion}

Staining with MDH reveals a maturation process in S. cerevisiae spore wall development as the spores become progressively impermeable to the dye over time. The basis for this process is unclear and the assay may be somewhat unique to $\mathrm{MDH}$ as spores were impermeable to a second lipid droplet dye, BODIPY-TR [14], as soon as they formed (K.Z., unpublished observations). Nonetheless, MDH staining provides a sensitive assay that allows differentiation of the phenotypes of genes affecting outer spore wall assembly.

The fact that dit1 $\Delta$ mutants develop impermeability like wild-type cells indicates that the dityrosine layer is dispensable for this property of the wall. This means that the MDH assay can distinguish whether mutants defective in dityrosine assembly are solely involved in formation of the dityrosine layer or if they have additional roles in wall assembly, suggesting their effects on dityrosine may be more indirect. The latter is certainly the case for mutation of NPP1/NPP2. Although earlier biochemical studies had suggested that reduction in dityrosine was the only major change in the outer spore wall in an npp1 $n p p 2 \Delta$ strain [14], the permeability of these spores to MDH clearly indicates additional abnormalities in the wall. Similarly, the osw $3 \Delta, \operatorname{osw} 4 \Delta \operatorname{osw} 6 \Delta$, and osw $7 \Delta$ she $10 \Delta$ mutant strains show intermediate levels of permeability suggesting roles beyond dityrosine assembly. Consistent with this, the osw $4 \Delta$ osw $6 \Delta$ and osw $7 \Delta$ she $10 \Delta$ mutants are more ether sensitive than a dit $1 \Delta$ mutant [14]. Whether the intermediate level of permeability in these mutants indicates that they fail to make some component of the wall or if they have a temporal delay in assembly remains to be determined. Deletions of OSW5 display somewhat reduced dityrosine fluorescence [17] and impermeability to $\mathrm{MDH}$, which may indicate that OSW5 is specifically involved in generation of the dityrosine layer.

In the mutants examined, there is a good correlation between ether resistance and impermeability to MDH staining, though it is not yet clear how these two properties of the outer spore wall are related. The two phenotypes are not perfectly linked as wild-type strains are resistant to ether even when the spores are newly formed and permeable to the dye. It may be that some component of the spore wall confers resistance to ether immediately upon deposition in the wall and then subsequently 'matures' to confer impermeability to MDH. There is also a correlation between the absence of Chi and ether sensitivity [14], suggesting the hypothetical component conferring ether resistance and impermeability could be Chi. However, this correspondence is not perfect as mutants like $n p p 1 \Delta n p p 2 \Delta$ are ether sensitive and permeable to MDH, but still have Chi present in their spore walls (Figure 3; [14]).

The OSW2 gene is particularly interesting in regards to the spore wall component responsible for impermeability. Mutants in OSW2 have no obvious cytological or biochemical defect in the spore wall and yet $o s w 2 \Delta$ spores are highly ether sensitive and permeable to MDH [18]. This suggests that OSW2 may play a relatively direct role in promoting these properties of the spore wall. The Osw2 protein is localized to the spore cytoplasm and has homology to ketopantoate reductase and the larger family of NADP dependent dehydrogenase enzymes ([31]; unpublished obs.). Therefore, rather than being directly involved in spore wall assembly, Osw2 could be involved in synthesis of some precursor that is exported from the cytoplasm to the wall similar to dityrosine. Consistent with the idea of export, the $q d r 1 \Delta q d r 3 \Delta d t r 1 \Delta$ strain deficient in multiple transporters [14] displays permeability, suggesting these transporters could be involved in export of the hypothetical Osw2 product. 
The phenotypes of the tyr $1 \Delta$ mutant demonstrate that de novo synthesis of tyrosine provides material for assembly of the dityrosine layer of the spore wall. Mutants in TYR1 do not have as severe an effect on dityrosine fluorescence as mutations in DIT1 indicating that much of the tyrosine for dityrosine synthesis must come from other sources. However, it is noteworthy that de novo tyrosine synthesis contributes to the spore wall as sporulation occurs under conditions of nitrogen starvation. This suggests that during sporulation, not only carbon atoms but also scarce nitrogen resources are shunted into the aromatic amino acid biosynthesis pathway to allow for spore wall formation.

In addition to providing tyrosine for dityrosine, the permeability of tyr1 $\Delta$ mutants to MDH indicates additional roles for tyrosine in spore wall synthesis. Given the permeability and ether sensitivity of the tyr $1 \Delta$ mutant, it is tempting to speculate that tyrosine could be a precursor to Chi or some other component made in an OSW2-dependent pathway. The ${ }^{13} \mathrm{C}$ NMR spectra by which Chi was defined do not include chemical shifts consistent with tyrosine [14], so if tyrosine is a precursor for Chi synthesis, it must be significantly modified. Nonetheless, our results expand the roles of aromatic amino acids in promoting fungal cell wall synthesis.

While spore wall organization is best studied in S. cerevisiae; the organization of the wall is likely similar in all ascomycetes. For example, the spore wall of the distantly related fission yeast Schizosaccharomyces pombe also has additional layers relative to the vegetative wall and the S. pombe spore wall, like the S. cerevisiae spore wall, uniquely contains chitosan [32-34]. Moreover, the sporulation-induced $m u g 72$ gene of $S$. pombe encodes an enzyme related to OSW2 [35], raising the possibility that the OSW2-generated component of the S. cerevisiae spore wall is present in the S. pombe wall as well. The outer surface of the $S$. pombe wall is constructed from a protein rather than dityrosine [36]; however, many other ascomycete genomes carry orthologs to the dityrosine synthesis genes DIT1 and DIT2 as well as the dityrosine exporter DTR1 and dityrosine has been detected in several other yeasts $[37,38]$. Thus, the mechanisms of assembly and properties of the spore wall in S. cerevisiae are likely to be a useful model for ascospore walls in other ascomycetes.

Acknowledgments: The authors wish to thank Carey Kim for plasmids and members of the Neiman Lab for helpful discussions. This work was supported by NIH grant R01 GM072540 to A.M.N.

Author Contributions: Aaron M. Neiman and Leor Needleman conceived and designed the experiments; Kai Zhang, Leor Needleman and Sai Zhou performed the experiments; Kai Zhang, Leor Needleman, Sai Zhou and Aaron M. Neiman analyzed the data; Aaron M. Neiman wrote the manuscript.

Conflicts of Interest: The authors declare no conflict of interest. The funding sponsors had no role in the design of the study; in collection, analyses, or interpretation of data, in writing of the manuscript; and in the decision to publish the results.

\section{References}

1. Carlile, M.J.; Watkinson, S.C. The Fungi, 4th ed.; Academic Press: San Diego, CA, USA, 1994.

2. Neiman, A.M. Sporulation in the budding yeast Saccharomyces cerevisiae. Genetics 2011, 189, 737-765. [CrossRef] [PubMed]

3. Briza, P.; Ellinger, A.; Winkler, G.; Breitenbach, M. Chemical composition of the yeast ascospore wall-The second outer layer consists of chitosan. J. Biol. Chem. 1988, 263, 11569-11574. [PubMed]

4. Briza, P.; Winkler, G.; Kalchhauser, H.; Breitenbach, M. Dityrosine is a prominent component of the yeast ascospore wall-A proof of its structure. J. Biol. Chem. 1986, 261, 4288-4294. [PubMed]

5. Lynn, R.R.; Magee, P.T. Development of the spore wall during ascospore formation in Saccharomyces cerevisiae. J. Cell Biol. 1970, 44, 688-692. [CrossRef] [PubMed]

6. Christodoulidou, A.; Bouriotis, V.; Thireos, G. Two sporulation-specific chitin deacetylase-encoding genes are required for the ascospore wall rigidity of Saccharomyces cerevisiae. J. Biol. Chem. 1996, 271, 31420-31425. [CrossRef] [PubMed]

7. Christodoulidou, A.; Briza, P.; Ellinger, A.; Bouriotis, V. Yeast ascospore wall assembly requires two chitin deacetylase isozymes. FEBS Lett. 1999, 460, 275-279. [CrossRef] 
8. Pammer, M.; Briza, P.; Ellinger, A.; Schuster, T.; Stucka, R.; Feldmann, H.; Breitenbach, M. Dit101 (csd2, cal1), a cell cycle-regulated yeast gene required for synthesis of chitin in cell walls and chitosan in spore walls. Yeast 1992, 8, 1089-1099. [CrossRef] [PubMed]

9. Briza, P.; Ellinger, A.; Winkler, G.; Breitenbach, M. Characterization of a dl-dityrosine-containing macromolecule from yeast ascospore walls. J. Biol. Chem. 1990, 265, 15118-15123. [PubMed]

10. Briza, P.; Kalchhauser, H.; Pittenauer, E.; Allmaier, G.; Breitenbach, M. N,N'-bisformyl dityrosine is an in vivo precursor of the yeast ascospore wall. Eur. J. Biochem. 1996, 239, 124-131. [CrossRef] [PubMed]

11. Briza, P.; Eckerstorfer, M.; Breitenbach, M. The sporulation-specific enzymes encoded by the DIT1 and DIT2 genes catalyze a two-step reaction leading to a soluble ll-dityrosine-containing precursor of the yeast spore wall. Proc. Natl. Acad. Sci. USA 1994, 91, 4524-4528. [CrossRef] [PubMed]

12. Felder, T.; Bogengruber, E.; Tenreiro, S.; Ellinger, A.; Sa-Correia, I.; Briza, P. Dtrlp, a multidrug resistance transporter of the major facilitator superfamily, plays an essential role in spore wall maturation in Saccharomyces cerevisiae. Eukaryot. Cell 2002, 1, 799-810. [CrossRef] [PubMed]

13. Eisenman, H.C.; Casadevall, A. Synthesis and assembly of fungal melanin. Appl. Microbiol. Biotechnol. 2012, 93, 931-940. [CrossRef] [PubMed]

14. Lin, C.P.; Kim, C.; Smith, S.O.; Neiman, A.M. A highly redundant gene network controls assembly of the outer spore wall in S. cerevisiae. PLoS Genet. 2013, 9, e1003700. [CrossRef] [PubMed]

15. Coluccio, A.E.; Rodriguez, R.K.; Kernan, M.J.; Neiman, A.M. The yeast spore wall enables spores to survive passage through the digestive tract of Drosophila. PLoS ONE 2008, 3, e2873. [CrossRef] [PubMed]

16. Dawes, I.W.; Hardie, I.D. Selective killing of vegetative cells in sporulated yeast cultures by exposure to diethyl ether. Mol. Gen. Genet. 1974, 131, 281-289. [CrossRef] [PubMed]

17. Suda, Y.; Rodriguez, R.K.; Coluccio, A.E.; Neiman, A.M. A screen for spore wall permeability mutants identifies a secreted protease required for proper spore wall assembly. PLoS ONE 2009, 4, e7184. [CrossRef] [PubMed]

18. Coluccio, A.; Bogengruber, E.; Conrad, M.N.; Dresser, M.E.; Briza, P.; Neiman, A.M. Morphogenetic pathway of spore wall assembly in Saccharomyces cerevisiae. Eukaryot. Cell 2004, 3, 1464-1475. [CrossRef] [PubMed]

19. Rose, M.D.; Fink, G.R. Methods in Yeast Genetics; Cold Spring Harbor Laboratory Press: Cold Spring Harbor, NY, USA, 1990.

20. Sikorski, R.S.; Hieter, P. A system of shuttle vectors and yeast host strains designed for efficient manipulation of DNA in Saccharomyces cerevisiae. Genetics 1989, 122, 19-27. [PubMed]

21. Huh, W.K.; Falvo, J.V.; Gerke, L.C.; Carroll, A.S.; Howson, R.W.; Weissman, J.S.; O'Shea, E.K. Global analysis of protein localization in budding yeast. Nature 2003, 425, 686-691. [CrossRef] [PubMed]

22. Goldstein, A.L.; McCusker, J.H. Three new dominant drug resistance cassettes for gene disruption in Saccharomyces cerevisiae. Yeast 1999, 15, 1541-1553. [CrossRef]

23. Longtine, M.S.; McKenzie, A., 3rd; Demarini, D.J.; Shah, N.G.; Wach, A.; Brachat, A.; Philippsen, P.; Pringle, J.R. Additional modules for versatile and economical PCR-based gene deletion and modification in saccharomyces cerevisiae. Yeast 1998, 14, 953-961. [CrossRef]

24. Neiman, A.M.; Katz, L.; Brennwald, P.J. Identification of domains required for developmentally regulated SNARE function in Saccharomyces cerevisiae. Genetics 2000, 155, 1643-1655. [PubMed]

25. Coluccio, A.; Neiman, A.M. Interspore bridges: A new feature of the Saccharomyces cerevisiae spore wall. Microbiology 2004, 150, 3189-3196. [CrossRef] [PubMed]

26. Rabitsch, K.P.; Toth, A.; Galova, M.; Schleiffer, A.; Schaffner, G.; Aigner, E.; Rupp, C.; Penkner, A.M.; Moreno-Borchart, A.C.; Primig, M.; et al. A screen for genes required for meiosis and spore formation based on whole-genome expression. Curr. Biol. 2001, 11, 1001-1009. [CrossRef]

27. Currie, E.; Guo, X.; Christiano, R.; Chitraju, C.; Kory, N.; Harrison, K.; Haas, J.; Walther, T.C.; Farese, R.V., Jr. High confidence proteomic analysis of yeast LDs identifies additional droplet proteins and reveals connections to dolichol synthesis and sterol acetylation. J. Lipid Res. 2014, 55, 1465-1477. [CrossRef] [PubMed]

28. Ren, J.; Pei-Chen Lin, C.; Pathak, M.C.; Temple, B.R.; Nile, A.H.; Mousley, C.J.; Duncan, M.C.; Eckert, D.M.; Leiker, T.J.; Ivanova, P.T.; et al. A phosphatidylinositol transfer protein integrates phosphoinositide signaling with lipid droplet metabolism to regulate a developmental program of nutrient stress-induced membrane biogenesis. Mol. Biol. Cell. 2014, 25, 712-727. [CrossRef] [PubMed] 
29. Briza, P.; Breitenbach, M.; Ellinger, A.; Segall, J. Isolation of two developmentally regulated genes involved in spore wall maturation in Saccharomyces cerevisiae. Genes Dev. 1990, 4, 1775-1789. [CrossRef] [PubMed]

30. Walther, T.; Letisse, F.; Peyriga, L.; Alkim, C.; Liu, Y.; Lardenois, A.; Martin-Yken, H.; Portais, J.C.; Primig, M.; Francois, J. Developmental stage dependent metabolic regulation during meiotic differentiation in budding yeast. BMC Biol. 2014, 12, 60. [CrossRef] [PubMed]

31. Lam, C.; Santore, E.; Lavoie, E.; Needleman, L.; Fiacco, N.; Kim, C.; Neiman, A.M. A visual screen of protein localization during sporulation identifies new components of prospore membrane-associated complexes in budding yeast. Eukaryot. Cell 2014, 13, 383-391. [CrossRef] [PubMed]

32. Arellano, M.; Cartagena-Lirola, H.; Nasser Hajibagheri, M.A.; Duran, A.; Henar Valdivieso, M. Proper ascospore maturation requires the chs1+ chitin synthase gene in Schizosaccharomyces pombe. Mol. Microbiol. 2000, 35, 79-89. [CrossRef] [PubMed]

33. Matsuo, Y.; Tanaka, K.; Matsuda, H.; Kawamukai, M. cda1+, encoding chitin deacetylase is required for proper spore formation in Schizosaccharomyces pombe. FEBS Lett. 2005, 579, 2737-2743. [CrossRef] [PubMed]

34. Matsuo, Y.; Tanaka, K.; Nakagawa, T.; Matsuda, H.; Kawamukai, M. Genetic analysis of chs1+ and chs2+ encoding chitin synthases from Schizosaccharomyces pombe. Biosci. Biotechnol. Biochem. 2004, 68, 1489-1499. [CrossRef] [PubMed]

35. Martin-Castellanos, C.; Blanco, M.; Rozalen, A.E.; Perez-Hidalgo, L.; Garcia, A.I.; Conde, F.; Mata, J.; Ellermeier, C.; Davis, L.; San-Segundo, P.; et al. A large-scale screen in S. pombe identifies seven novel genes required for critical meiotic events. Curr. Biol. 2005, 15, 2056-2062. [CrossRef] [PubMed]

36. Fukunishi, K.; Miyakubi, K.; Hatanaka, M.; Otsuru, N.; Hirata, A.; Shimoda, C.; Nakamura, T. The fission yeast spore is coated by a proteinaceous surface layer comprising mainly Isp3. Mol. Biol. Cell 2014, 25, 1549-1559. [CrossRef] [PubMed]

37. Smail, E.H.; Briza, P.; Panagos, A.; Berenfeld, L. Candida albicans cell walls contain the fluorescent cross-linking amino acid dityrosine. Infect. Immun. 1995, 63, 4078-4083. [PubMed]

38. Prillinger, H.; Schweigkofler, W.; Breitenbach, M.; Briza, P.; Staudacher, E.; Lopandic, K.; Molnar, O.; Weigang, F.; Ibl, M.; Ellinger, A. Phytopathogenic filamentous (Ashbya, Eremothecium) and dimorphic fungi (Holleya, Nematospora) with needle-shaped ascospores as new members within the Saccharomycetaceae. Yeast 1997, 13, 945-960. [CrossRef]

(c) 2017 by the authors. Licensee MDPI, Basel, Switzerland. This article is an open access article distributed under the terms and conditions of the Creative Commons Attribution (CC BY) license (http:/ / creativecommons.org/licenses/by/4.0/). 